\title{
ESTUDIO DE ALCANCE DE TERAPIA OCUPACIONAL Y DROGODEPENDENCIAS: REFLEXIONES CRÍTICAS DE LOS CONTEXTOS, PRIORIDADES Y MIRADAS EN LA INVESTIGACIÓN
}

\author{
A SCOPING REVIEW OF OCCUPATIONAL THERAPY LITERATURE ON DRUG \\ ADDICTION: CRITICAL REFLECTIONS ON CONTEXTS, PRIORITIES AND RESEARCH \\ PERSPECTIVES
}

\section{Lucía González ${ }^{1}$, Natalia Rivas-Quarneti² y Lisette Farías ${ }^{3}$}

\begin{abstract}
Resumen
Introducción: El consumo problemático de drogas representa uno de los problemas de salud más significativos a nivel mundial. La terapia ocupacional es uno de los actores sociales que promueve la participación en la comunidad, mediante la ocupación, contribuyendo a la justicia ocupacional. Sin embargo, se desconoce qué perspectivas y prioridades se están utilizando en la investigación de la terapia ocupacional, lo cual es clave para desarrollar una práctica reflexiva y crítica. Objetivo: Actualizar, identificar y sintetizar las prioridades y perspectivas en la producción científica existente relacionada al consumo de drogas y terapia ocupacional. Método: Se realizó una revisión de la literatura siguiendo el marco de referencia para "Scoping Review" desarrollado por Arksey y O’Malley. Se buscó en las siguientes bases de datos electrónicas: CINAHL, Cochrane Library Plus, Dialnet, EMBASE, ISI Web of Science, OTseeker y Scopus. Se empleó el método descriptivo-analítico y la triangulación de investigadores para realizar el análisis de los datos. Resultados: Del análisis de la literatura emergieron tres categorías: "Contextualización", "Prioridades de investigación" -relación entre el desempeño ocupacional y la calidad de vida, instrumentos de evaluación e intervenciones de terapia ocupacional- y "Miradas teóricas en la investigación" (una mirada positivista: la neurociencia como sustento de la intervención; una mirada empoderadora: construyendo el camino hacia la inclusión, y una mirada sistémica: centrada en la familia como base de la intervención). Conclusión: Es preciso continuar investigando acerca de la problemática del consumo de drogas desde la terapia ocupacional, empleando una perspectiva crítica basada en la ciencia de la ocupación.
\end{abstract}

\section{Palabras clave:}

drogodependencias, terapia ocupacional, estudio de alcance.

Fecha de recepción: 19/04/2016.

Fecha de aceptación: 27/10/2016.

1 Terapeuta Ocupacional, graduada en la Universidade da Coruña. Dirección: Xubias de Arriba, Campus de Oza (15006). Teléfono: +34616780317. Email: lucia.gboquete@udc.es.

2 Terapeuta Ocupacional. Profesora de la Facultad de Ciencias de la Salud, Universidade da Coruña. Doctora en Ciencias Sociosanitarias, Universidade da Coruña, España. Máster Europeo de Ciencias en Terapia Ocupacional. Dirección: Xubias de Arriba, Campus de Oza (15006). Teléfono: +34881015968. Email: natalia.rivas.quarneti@udc.es.

3 Candidata a Doctora en Ciencias de la Salud y Rehabilitación, Western University, Canadá. Máster Europeo de Ciencias en Terapia Ocupacional. Licenciada en Ciencia de la Ocupación. Terapeuta Ocupacional. Dirección: 662 Platt's Lane, London, Ontario, N6G 3B2, Canadá. Teléfono: +15192807496. Email: Ifariasv@uwo.ca. 


\begin{abstract}
Introduction: Drug abuse is one of the most complex global health issues. Occupational therapy is one of the social actors that promotes community participation, using occupation as a means to contribute to occupational justice. However, it is unknown what theoretical perspectives are being used in occupational therapy research. This knowledge is essential for developing a critical and reflexive practice. Objective: This study aims to update, identify and synthesize the priorities and perspectives employed in the existing scientific literature of occupational therapy related with problematic use of drugs. Method: A scoping literature review based on the methods described by Arksey and O'Malley (2005) was conducted. Literature was identified using the following electronic databases: CINAHL, Cochrane Library Plus, Dialnet, EMBASE, ISI Web of Science, OTseeker, and Scopus. To analyse the data, a descriptive-analytic method and triangulation were used. Results: The literature reviewed reveals information regarding three categories: "Contextualization", "Research Priorities" -the relationship between occupational performance and quality of life, assessment tools, occupational therapy intervention and "Theoretical Research Perspectives" (a positivist view: neuroscience as base for intervention, an empowering view: paving the way for inclusion, and a systemic view: family as base for intervention). Conclusion: Further research is needed regarding the role of occupational therapy in the field of problematic drug use using a critical perspective based on occupational science.
\end{abstract}

\title{
Keywords:
}

drug addiction, occupational therapy, scoping review.

\section{INTRODUCCIÓN}

El alcance del consumo de sustancias psicoactivas en todo el mundo se estima en 185 millones de usuarios (United Nations Office for Drug Control and Crime Prevention [UNDCP], 2002). Sin embargo, una de las diferencias entre las sustancias psicoactivas más conocidas (ej. tabaco, alcohol y drogas ilícitas) es el hecho de que el consumo de drogas ilícitas tiene un efecto más pronunciado en grupos más jóvenes, causando una morbilidad y/o discapacidad temprana en la vida (World Health Organization [WHO], 2002). Por ello, a nivel mundial se han llevado a cabo diversos esfuerzos para estimar los alcances del uso ilícito de drogas, ya que al ser un consumo ilícito y oculto es difícil hacer estimaciones exactas de cómo este comportamiento incrementa el número de muertes en el mundo (Degenhardt, Hall, Warner-Smith \& Lynskey, 2004). Por ello, el consumo problemático de drogas constituye uno de los problemas de salud más complejos de salud pública. Este fenómeno es de gran relevancia puesto que es un patrón de comportamiento que tiene un importante efecto negativo sobre la salud y el bienestar de las personas que participan en ella, produciendo una sustancial pérdida de la vida y discapacidad (Degenhardt, et al. 2004). En Europa, algunas de las principales consecuencias del consumo de drogas en la salud de los jóvenes se han relacionado con la pérdida de autonomía, problemas con la justicia, depresión, estrés o ansiedad (Observatorio Europeo de las Drogas y las Toxicomanías [EMCDDA], 2013), lo que hace necesaria una asistencia socio-sanitaria. Sin embargo, solo 82 países a nivel mundial ofrecen servicios a quienes sufren la problemática del consumo de drogas (Organización Mundial de la Salud [OMS], 2012).

A nivel nacional, es necesario comentar que en 2008 se inició en España la llamada "crisis económi$\mathrm{ca}^{\prime \prime}$ que se mantiene hasta la actualidad. Como consecuencia, según el Plan Nacional sobre Drogas 2013 - 2016, el consumo de drogas ha descendido en los últimos años. Sin embargo, el consumo de sustancias más económicas ha aumentado (ej. alcohol, cocaína, cannabis), al igual que la demanda asistencial de servicios destinados a la problemática del consumo de drogas. No obstante, este incremento en la demanda asistencial no ha obtenido una respuesta satisfactoria debido a una disminución de los recursos económicos destinados a estos servicios a nivel nacional (Ministerio de Sanidad y Política Social, 2012).

Desde la terapia ocupacional (TO) se ha evidenciado que el consumo problemático de drogas tiene 
una repercusión directa en el desempeño ocupacional de las personas y en su salud (De León, Cantero \& Zabala, 2013). Algunos ejemplos son el deterioro de los roles, la alteración de las rutinas diarias o el abandono de los intereses personales, lo que lleva a una disfunción ocupacional (De León, et al., 2013; Farías, Guerra, Cifuentes, Rozas \& Riveros, 2010). Sin embargo, a pesar de la magnitud y relevancia de la problemática, en Galicia (la comunidad autónoma de residencia de las dos primeras autoras), los planes de actuación socio-sanitaria carecen de la figura del terapeuta ocupacional como parte de los equipos de intervención en este ámbito (Consellería de Sanidade e Servicio Galego de Saúde [SERGAS], 2011). Paralelamente, la terapia ocupacional y la ciencia de la ocupación (CO) han vivido un avance exponencial en las últimas décadas, especialmente en lo referente al fortalecimiento del compromiso por la transformación social y el trabajo para y con poblaciones en situaciones de vulnerabilidad o exclusión, en las que suelen encontrarse personas que presentan consumo problemático de drogas (Kronenberg, Simo \& Pollard, 2007; Pollard, Sakellariou \& Kronenberg, 2008). Unido a esto, la necesidad de basar la práctica en la evidencia ha generado un gran interés de explorar la investigación realizada sobre la terapia ocupacional y el consumo problemático de sustancias desde una perspectiva crítica, para generar una discusión reflexiva acerca de los conocimientos actuales (ej. prioridades, perspectivas) de la TO en el área de drogodependencias. De esta forma, con la realización de esta revisión se pretende actualizar, identificar y sintetizar los conocimientos actuales y las aproximaciones de la TO en la problemática del consumo de drogas. Asimismo, se pretende facilitar la actualización teórica y práctica en este ámbito que pueda ser de utilidad para promover la inclusión y posicionamiento de la figura de la TO en esta área.

\section{MÉTODO}

Para explorar la producción científica de la TO en el área de las drogodependencias se realizó una revisión bibliográfica siguiendo el marco de referencia para "Scoping Reviews" propuesto por Arksey y O'Malley
(2005). Una scoping review tiene como finalidad profundizar en los conceptos teóricos clave que sustentan un área de investigación y las principales fuentes y tipos de evidencia disponibles. Este tipo de revisión de la literatura puede llevarse a cabo cuando un área es compleja o no ha sido revisada exhaustivamente con anterioridad. Asimismo, las scoping reviews se suelen utilizar para hace hincapié en la necesidad de ampliar la cobertura de la literatura disponible (Arksey \& O'Malley, 2005). Según Arksey y O'Malley (2005), las etapas para la realización de una scoping review son:

- Etapa 1: Identificación de la pregunta de investigación.

- Etapa 2: Identificación de los estudios relevantes.

- Etapa 3: Selección de los estudios.

- Etapa 4: Análisis de los datos.

- Etapa 5: Recopilación y resumen de los resultados.

En la etapa 1 se identificó como pregunta de investigación: ¿Cómo se describe el rol, prioridades y perspectivas de investigación en la literatura de TO relacionada con el consumo problemático de drogas? Para la identificación de estudios relevantes (etapa 2) se utilizaron las siguientes bases de datos electrónicos: CINAHL, Cochrane Library Plus, Dialnet, EMBASE, ISI Web of Science, OTseeker y Scopus. Para la selección de artículos para este estudio (etapa 3) se utilizaron una serie de criterios de inclusión y exclusión, los cuales se construyeron de acuerdo a las sugerencias propuestas por Arksey y O'Malley (2005) (Ver Tabla 1). Del total de literatura encontrada $(\mathrm{n}=291)$ se seleccionaron 21 estudios potencialmente adecuados para su lectura a texto completo, de los cuales 7 fueron excluidos por no cumplir los criterios de inclusión y exclusión. Finalmente, 14 artículos constituyen la muestra final de este estudio.

De acuerdo a los criterios de inclusión y exclusión se seleccionaron artículos originales en revistas científicas del ámbito socio-sanitario (ej. revisiones sistemáticas, investigaciones, abordajes y/o casos de estudio), en inglés y español, entre los años 2005 y 2015 (Ver Figura 1). 
Tabla 1:

Criterios de inclusión/exclusión.

\begin{tabular}{|c|c|}
\hline Criterios de inclusión & Criterios de exclusión \\
\hline Artículos originales en revistas científicas. & $\begin{array}{c}\text { Artículos de opinión, cartas al director, reseñas bibliográ- } \\
\text { ficas o artículos de colaboración. }\end{array}$ \\
\hline Publicaciones en inglés y español entre los años 2005 y 2015. & Revisiones de la literatura. \\
\hline
\end{tabular}

Figura 1:

Proceso de búsqueda en las bases de datos.

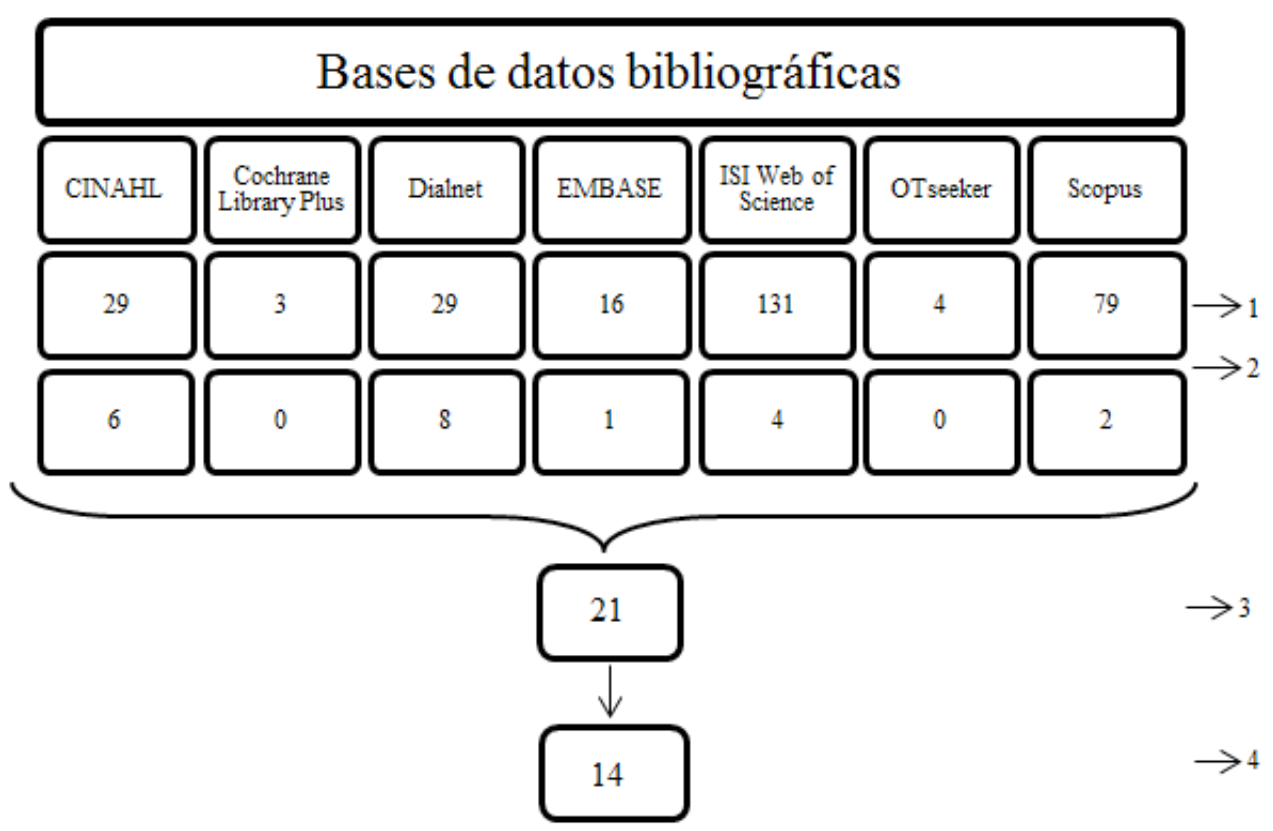

1. Artículos devueltos

2. Eliminación de duplicados/Cribado aplicando criterios de incl./excl.

3. Artículos analizados

4. Artículos seleccionados

El análisis de los datos (etapa 4) se realizó de manera continua y progresiva para construir un orden, estructura y significado del conjunto de datos recogidos. De esta manera el análisis se llevó a cabo de forma circular, es decir, se inicia desde el comienzo de la recogida de la información, lo cual implica un continuo análisis y revisión de la misma (González \& Cano, 2010). Además, se empleó un método descriptivo-analítico, que consiste en la aplicación de un marco analítico común a todos los estudios de investigación y la recopilación 
de la información más relevante de cada uno (Taylor \& Bogdan, 1990). El rigor metodológico se aseguró mediante distintos procedimientos, entre ellos la triangulación de investigadoras, en este caso las autoras, para reducir la posibilidad de sesgos sistemáticos (Law, Stewart, Lette, Pollock Bosch, \& Westmorland, 1998).

\section{Resultados}

Del análisis de los estudios incluidos en esta revisión emergieron 3 categorías relevantes: "Contextualización", "Prioridades en la investigación" y "Miradas teóricas en la investigación". A continuación se describirán cada una de ellas.

\section{Contextualización}

La categoría de contextualización de los estudios se estructura en: información de las/los autores, metodología y diseño del estudio, lugar y año de publicación y población de estudio.

En cuanto a los autores, 6 de los documentos fueron realizados exclusivamente por terapeutas ocupacionales (De León et al., 2013; Rojo, 2010; Rivas, Gómez, Movilla \& Viana, 2011; Farías et al., 2010; Colomer, 2008; Thompson, 2007), 6 por un equipo interdisciplinario en el que existe la figura del TO (Wasmuth, Crabtree \& Scott, 2014; Rojo, Pedrero, Ruiz, Llanero \& Puerta, 2011; Colomer, Cabellos \& Bustos, 2011; Rojo, Pedrero, Ruiz, Llanero, Puerta \& Olivar, 2009; Martin, Bliven \& Boisvert, 2008; Boisvert, Martin, Grosek \& Clarie, 2008) y 2 fueron llevados a cabo por profesionales de otras disciplinas relacionadas con la TO (Davies \& Cameron, 2010; Díaz \& Palucci, 2010).

En cuanto a la metodología de los estudios, 9 artículos emplean metodología de tipo cualitativa (Wasmuth et al., 2014; Rojo, 2010; Rivas et al., 2011; Díaz \& Palucci, 2010; Davies \& Cameron, 2010; Farías et al., 2010; Rojo et al., 2009; Martin et al., 2008; Thompson, 2007), 1 de tipo cuantitativa (Rojo et al., 2012) y 4 de tipo mixta (De León et al., 2013; Colomer et al., 2011; Colomer, 2008; Boisvert et al., 2008). En cuanto a la metodología cualitativa y mixta, la mayoría de estos estudios definen el diseño de estudio como estudios descriptivos transversales $(n=5)$ (De León et al., 2013; Colomer et al., 2011; Díaz \& Palucci, 2010; Boisvert et al., 2008; Martin et al., 2008), estudios narrativos $(n=2)$ (Wasmuth et al., 2014, Davies
\& Cameron, 2010), estudios exploratorios transversales $(n=2)$ (Farías et al., 2010; Thompson, 2007) e investigación y acción participación ( $n=1$ ) (Rivas et al., 2011). En cuanto a la metodología cuantitativa se ha realizado un ensayo clínico ( $n=1$ ) (Rojo et al., 2011). Además, hay algunos artículos que se definen como propuestas teóricas $(n=3)$ (Rojo, 2010; Rojo et al., 2009; Colomer, 2008).

En lo referente al lugar de publicación, se encontró que la mayoría de estos artículos proceden de España $(n=7)$ (De León et al., 2013; Rojo, 2010; Colomer et al., 2011; Rivas et al., 2011; Rojo et al., 2011; Rojo et al., 2009; Colomer, 2008). Asimismo, algunos estudios se realizaron en Estados Unidos $(n=3)$ (Boisvert et al., 2008; Martin et al., 2008; Thompson, 2007), Reino Unido $(\mathrm{n}=2)$ (Wasmuth, 2014; Davies \& Cameron, 2010), Chile $(n=1)$ (Farías et al., 2010) y Brasil $(n=1)$ (Díaz \& Palucci, 2010).

Respecto a los años de publicación se ha encontrado que los más productivos han sido el 2008 (Boisvert et al, 2008; Colomer, 2008; Martin et al., 2008), 2010 (Farías et al., 2010; Díaz \& Palucci, 2010; Davies \& Cameron; 2010) y 2011 (Rojo et al., 2011; Colomer et al., 2011; Rivas et al., 2011), con 3 publicaciones en cada uno.

En cuanto a la población, coherentemente con los criterios de inclusión, todos los documentos hacen referencia a personas con consumo problemático de drogas, sin embargo existe una gran diversidad en la manera de entenderla ( $n=6)$ (Wasmuth, 2014; De León et al., 2013; Rojo at al., 2011; Davies \& Cameron, 2010; Rojo et al., 2009; Martin et al., 2008). Así, 2 artículos incluyen específicamente a personas con consumo problemático de alcohol en este grupo (Rojo, 2010; Colomer et al., 2011). En uno de los estudios, además de la situación de drogodependencia, los participantes se encontraban en situación de calle (Boisvert, 2008). Otras investigaciones $(n=2)$ (Rivas et al., 2011; Colomer, 2008) describen e incluyen a participantes con distintas características asociadas a la drogodependencia, por ejemplo personas con consumo problemático de alcohol, personas en situación de calle, en riesgo y/o situación de exclusión social, población reclusa o menores de edad. Por otro lado, en 3 de los artículos los participantes eran terapeutas ocupacionales que trabajaban en el ámbito de las drogodependencias (Farías et al., 2010; Díaz \& Palucci, 2010; Thompson, 2007).

\section{Prioridades en la investigación}

En la categoría "Prioridades en la investigación" se distinguen 3 inquietudes que los investigadores han 
aproximado en sus estudios. A continuación se elaborará cada una de ellas.

a) Relación entre el desempeño ocupacional y la calidad de vida

Los artículos analizados (De León et al., 2008; Colomer et al., 2011; Martin et al., 2008) señalan como una prioridad de la investigación la "relación entre el desempeño ocupacional y la calidad de vida". Este interés se aprecia en los estudios que, por ejemplo, han empleado: el cuestionario Auto-informe de Desempeño Ocupacional (ADO) (De León et al., 2008), la Escala de Evaluación de Actividades de la Vida Diaria en Adicciones del CAD-1 (Colomer et al., 2011) y Occupational Performance History Interview (Martin et al., 2008). Estas evaluaciones en general permiten realizar una evaluación del desempeño ocupacional de las personas y observar cómo es la importancia del entorno según el valor que le otorguen a cada actividad de la vida diaria (AVD). Por ejemplo, De León et al. (2013) ofrecen una descripción del impacto que tiene el consumo problemático de drogas en el desempeño ocupacional de las personas, teniendo en cuenta las actividades de la vida diaria, trabajo, ocio y tiempo libre. En cuanto a las $A V D$, señalan que puede existir un compromiso de las habilidades de la persona para realizar sus actividades de autocuidado, presentando mayores alteraciones en las actividades básicas de la vida diaria (ABVD), por ejemplo alimentación, descanso y sueño, y aseo e higiene personal (De León et al., 2013; Colomer et al., 2011), y en las actividades instrumentales de la vida diaria (AIVD), por ejemplo el cuidado del espacio personal, preparación de la comida y manejo del dinero y del tiempo (De León et al., 2013).

Del mismo modo, Colomer et al. (2011) y Martin et al. (2008) muestran estos hallazgos en sus resultados. Respecto al área de trabajo, estos estudios señalan que el efecto de la dependencia a las sustancias sobre el desempeño laboral de la persona implica importantes aspectos negativos al asociarse con la disminución de la productividad, el ausentismo y los accidentes, entre otros. En lo referente a las actividades de ocio y tiempo libre, De León et al. (2013) plantean que las personas con consumo problemático de drogas comúnmente experimentan una pérdida de intereses. Asimismo, Martin et al. (2008) también reflejan esta idea en su investigación, señalando que la mayoría de los intereses de las personas con consumo problemático de drogas se asocian con el uso y adquisición de la sustancia.
Además, se puede observar que las áreas del desempeño ocupacional que más se ven comprometidas son las que se le otorga un menor nivel de importancia (De León et al., 2013). Un ejemplo es el manejo del dinero, que se manifiesta como principal dificultad entre las personas con consumo problemático de drogas, y a su vez como la actividad que menor importancia le atribuyen a este grupo (De León et al., 2013). Por otro lado, entre las actividades con más potencial de trabajo terapéutico se hallan el manejo de sus necesidades básicas y el mantenimiento de buenas relaciones sociales, las cuales son percibidas con una estrecha relación con sus intereses principales.

En resumen, los artículos analizados (De León et al., 2008; Colomer et al., 2011; Martin et al., 2008) señalan como una prioridad de la investigación la relación entre el desempeño ocupacional y la calidad de vida, ya que como se ha comprobado, el abuso problemático de drogas afecta la salud, el bienestar y la calidad de vida de las personas. De León et al. (2013) ejemplifican así esta relación significativa: "las personas se perciben con mejor calidad de vida cuanto mejor es su desempeño ocupacional" (p. 18).

\section{b. Instrumentos de evaluación}

Varios autores (Rojo, 2010; Rojo et al., 2011; Farías et al., 2010; Thompson, 2007) plantean la necesidad de diseñar modelos, instrumentos de evaluación, propuestas de intervención y enfoques específicos del ámbito de las drogodependencias desde la TO. Además, se expone que teniendo modelos e instrumentos propios de la profesión en esta área, la justificación teórica de la disciplina será más evidente, promoviendo así intervenciones que busquen dar significado y propósito a las ocupaciones de las personas con consumo problemático de drogas.

En cuanto a la evaluación de TO en el área de adicciones, se identificaron propuestas provenientes, principalmente, de España y Chile. En España se plantean diversas líneas de evaluación para el tratamiento de la problemática del consumo de drogas que constan especialmente de: entrevistas semiestructuradas, observación participante, Índice de Barthel, Escala de Lawton y Brody, Cuestionario de Calidad de Vida CAD-4, escalas MEDLS y KELS y Escala de Autoestima de Rosenberg, entre otros (De León et al., 2013; Rojo et al., 2012; Colomer et al., 2011; Rojo et al., 2011; Colomer, 2008). Además, estos artículos han evidenciado una ausencia de instrumentos de evaluación específicos destinados a 
determinar los componentes ocupacionales relacionados con el consumo problemático de drogas (De León et al., 2013; Farías et al., 2010). Por ello, Rojo et al. (2011) propusieron diseñar el Auto-informe de Desempeño Ocupacional (ADO), un cuestionario adaptado del Occupational Self Assessment (OSA) de Kielhofner (2008), y el Cuestionario "Disejecutivo" (DEX-Sp), validados específicamente para población con consumo problemático de drogas, los cuales permiten evaluar la autopercepción de la calidad del desempeño ocupacional y de la influencia del ambiente en el desarroIlo de las AVD. De este modo, los autores se plantean un protocolo de evaluación basado en el Modelo de Rehabilitación Cognitiva Funcional de Lee, Powell \& Esdaile (2001). Asimismo, se propone un protocolo para la evaluación neuropsicológica de las adicciones, que se compone de test neuropsicológicos, escalas de sintomatología cotidiana y pruebas de desempeño ocupacional. Específicamente en España se validó la Evaluación Cognitiva de Montreal (MOCA) para la detección de alteraciones en las funciones neuro-cognitivas en personas con consumo problemático de sustancias (Rojo et al., 2012). Según Rojo y colegas (2011), actualmente existen instrumentos que permiten evaluar la calidad del desempeño ocupacional de las personas con adicción a sustancias en condiciones de gran validez ecológica. Sin embargo, estas evaluaciones de carácter observacional deben ser completadas con la propia perspectiva de la persona, puesto que no es concebible establecer los objetivos de un plan de intervención terapéutico sin contar con los propios intereses y valores de las personas implicadas, si lo que se pretende es conseguir un impacto significativo en su vida.

Por otra parte, en un estudio realizado en Chile (Farías et al., 2010) se exploraron los componentes ocupacionales que los terapeutas ocupacionales chilenos evalúan durante el proceso de tratamiento del consumo de drogas. Los resultados señalaron que la mayoría de los profesionales evalúan los componentes de rutina, hábitos, roles, volición, tiempo libre e historia laboral en la etapa de ingreso en centros de tratamiento y rehabilitación. Asimismo, se revela una carencia de modelos y evaluaciones adecuados y específicos desde la TO en el área de consumo problemático de drogas. Además, este estudio pone de manifiesto un uso elevado de modelos no propios de la disciplina. Así, el modelo comúnmente empleado por todos los entrevistados fue el Modelo de Ocupación Humana (MOHO). Sin embargo, las autoras señalan que la diversidad de enfoques teóricos que poseen los profesionales en esta área, no asegura un correcto uso de su terminología (Farías et al., 2010).

Sumado a lo anterior, las autoras sugieren que el entendimiento entre distintos terapeutas ocupacionales se ve entorpecido debido a la gran variabilidad en la terminología utilizada para hacer referencia a los aspectos ocupacionales afectados por el consumo de drogas. Por lo tanto, el estudio conducido por Farías et al. (2010) muestra la necesidad de unificar criterios y poseer un lenguaje común que facilite la comunicación y el intercambio de experiencias y permita elaborar registros e investigaciones basados en un mismo sustento. En la misma línea, Thompson (2007) pone de manifiesto la necesidad de incorporar la evaluación y el tratamiento del consumo problemático de sustancias y de las alteraciones que comprometen el desempeño ocupacional en la práctica diaria para asegurar un tratamiento integral.

c. Descripción de las intervenciones de terapia ocupacional y sus resultados

En los artículos analizados se presenta como una prioridad describir las diferentes intervenciones llevadas a cabo desde la TO y sus resultados más relevantes. Para aproximar esta prioridad, se describirán los contextos de las intervenciones.

Primero, existe una gran diversidad de contextos (ej. asociaciones, centros de atención a la drogodependencia, comunidades terapéuticas, unidades de día, etc.) desde los cuales se aborda el proceso de intervención. Segundo, en la mayor parte de los estudios, la intervención realizada desde la TO se centra en el desempeño ocupacional, tanto en las ABVD como en las AIVD, seguido por las áreas ocupacionales de participación social, ocio y tiempo libre (Wasmuth, 2014; De León et al., 2013; Colomer, 2008; Martin et al., 2008). Tercero, los resultados obtenidos en las intervenciones de TO generalmente son positivos, observando un aumento en los ítems estudiados (Colomer et al., 2011; Rivas et al., 2011; Davies \& Cameron, 2010; Martin et al., 2008; Boisvert et al., 2008). Por ejemplo, en el artículo de Martin et al. (2008) se describe una mejoría funcional a los 6 meses de las personas con consumo problemático de drogas que participaron en el proceso de recuperación. En tanto, Boisvert et al. (2008) señalan en su estudio una reducción significativa del riesgo de recaídas gracias a los grupos de apoyo entre pares. En estos casos se puede afirmar que la intervención de TO favorece la consecución de la autonomía personal, la participación activa en la comunidad, la percepción de 
la calidad de vida, la influencia de los entornos y contextos significativos y la reducción del riesgo de recaída. Además, estos estudios (Wasmuth, 2014; De León et al., 2013; Colomer, 2008; Martin et al., 2008) plantean una intervención terapéutica desde la perspectiva ocupacional para personas con consumo problemático de sustancias.

En contraposición, otras investigaciones (Rojo, 2010; Rojo et al., 2009) ponen de manifiesto el papel que la TO puede desarrollar en relación a los más recientes modelos neuropsicológicos de la adicción, que tratan de explorar las alteraciones debidas a la disfunción ejecutiva. Además, en cuanto a la descripción de las intervenciones, algunos autores hallaron que existen contradicciones entre la teoría y la práctica de la TO en drogodependencias. En los artículos de Wasmuth (2014), De León et al. (2013) y Colomer (2008) se señala que parece haber un acuerdo en la concepción de la TO y las funciones que debería tener el terapeuta ocupacional a nivel teórico. Sin embargo, cuando se pone en práctica, la TO adopta formas muy diversas y que incluso parecen contradictorias (Farías et al., 2010; Díaz \& Palucci, 2010; Thompson, 2007). Estas incongruencias son subrayadas por Díaz y Palucci (2010), quienes realizaron un estudio referente al papel de los diferentes profesionales que trabajan en centros de atención al consumo problemático de drogas en Bogotá. En sus resultados encontraron que los terapeutas ocupacionales son quienes realizaban talleres, actividades grupales, consultas individuales, actividades lúdicas y de prevención, actividades asistenciales y administrativas, y promovían las relaciones interpersonales en la comunidad.

En resumen, las intervenciones y contextos en donde se realiza terapia ocupacional son variados, al igual que los resultados de estas intervenciones, lo que indica que la TO presenta incoherencias entre su función definida teóricamente (área de estudio y formación) y su práctica. Algunas de las intervenciones que se esperaba encontrar en esta área pero no se hallaron en los artículos son las relacionadas a inclusión social, comunitaria y laboral (Thompson, 2007).

\section{Miradas teóricas en la investigación}

La categoría "Miradas teóricas en la investigación" hace referencia a las perspectivas de TO subyacentes en la aproximación a la investigación en el ámbito de las drogodependencias. A continuación se describirán cada una de ellas: a) Una mirada positivista: la neurociencia como sustento de la intervención

El aporte de la TO se ha evidenciado en propuestas como la intervención en la rehabilitación de la disfunción ejecutiva en personas con consumo problemático de drogas (Rojo et al., 2009) y en la evaluación del desempeño ocupacional "basada en la función, centrada en las actividades de la vida diaria desde los aspectos globales del comportamiento hasta los sustratos neurológicos y neuropsicológicos que lo sustentan" (Rojo, 2010, p. 33). Bajo esta mirada positivista desde las neurociencias, la adicción se considera como una alteración del funcionamiento cerebral independiente del ambiente y/o influencia social. Según los artículos revisados, este enfoque ofrece la posibilidad de entrenar las funciones alteradas que llevaron a la adicción y la mantuvieron en el tiempo, de modo que el resto de intervenciones puedan amplificar sus efectos e incrementar su efectividad (Rojo et al., 2009). Basado en este enfoque, Rojo y colegas (2009) proponen un protocolo de intervención desde TO que pueda ser incluido en los programas interdisciplinares de tratamiento de las adicciones. Rojo y colegas (2009) además proponen 3 niveles de actuación en la etapa de rehabilitación: actividades y métodos habilitadores, actividades propositivas y ocupaciones.

En resumen, en los artículos de Rojo (2012) y Rojo y colegas (2009), la problemática del consumo de drogas se plantea desde el punto de vista de las neurociencias, el cual se centra en los mecanismos neurobiológicos que intervienen en el inicio, mantenimiento y recaídas de las adicciones, enfocándose en las funciones ejecutivas alteradas por el consumo de drogas y resaltando la labor del terapeuta ocupacional en este campo. Así, Rojo y colegas (2009) sostienen que la experiencia acumulada por la TO con personas con daño cerebral adquirido, particularmente en la evaluación y tratamiento de las disfunciones ejecutivas, puede facilitar la comprensión y aportar una intervención específica de las alteraciones neurológicas en personas con consumo problemático de drogas, y su tratamiento, ya sea favoreciendo la adaptación o restauración de las funciones alteradas, a partir de la habilitación de los sustratos cerebrales alterados.

b) Una mirada empoderadora: construyendo el camino hacia la inclusión

Algunas de las investigaciones enfatizan el rol de la TO en el abordaje de la problemática del consumo de drogas desde diversas miradas teóricas. Por ejemplo, 
los resultados obtenidos en la investigación de Rivas et al. (2011) evidenciaron cómo las nuevas tecnologías de la información y la comunicación (TIC), una ocupación cultural y socialmente significativa, utilizadas desde un prisma emancipador, actúan como promotoras de la participación e inclusión social. Asimismo, estos resultados constatan el empoderamiento de la población con la que se trabajó e incluso de sus círculos cercanos, construyendo el proceso a través del reconocimiento y la redistribución, la reflexión, el razonamiento y las acciones emancipadoras de los actores involucrados.

Los resultados del estudio de Rivas et al. (2011) confirmaron no solo la responsabilidad que posee la comunidad de ofrecer oportunidades para participar, así como de fomentar el empoderamiento de los ciudadanos generando una comunidad más inclusiva, sino también los principios de la disciplina de la TO, que conciben la ocupación como una herramienta con un alto potencial para la inclusión social. Además, las autoras Rivas et al. (2011) concluyen que a través del proceso de construcción conjunta se fueron alcanzando los pequeños logros progresivamente, optimizando los recursos existentes para generar una comunidad más inclusiva. Con esta mirada, también Boisvert et al. (2008) en su estudio informan que la intervención comunitaria de TO de apoyo entre pares puede contribuir a la disminución de las recaídas en el consumo. Paralelamente, se observó un impacto positivo significativo en la recuperación de las adicciones a sustancias y la situación de calle. Este tipo de abordaje se ha venido reclamando desde hace años, como lo demuestran Colomer (2008), Martin et al. (2008) y Thompson (2007) en sus publicaciones.

También, el estudio de Davies \& Cameron (2010) señala que identificar las potencialidades y prioridades favorecerá la reinserción laboral de las personas con problemas de abuso de drogas. Por ejemplo, en esta investigación los participantes refieren que el consumo de drogas les había impedido lograr sus metas a nivel profesional. Esta afirmación es reforzada por los resultados obtenidos tanto de la Autoevaluación Ocupacional - OSA (Rojo et al., 2011; David \& Cameron, 2010) como de las entrevistas semiestructuradas (Wasmuth et al., 2014; Colomer, 2008; Boisvert et al., 2008), que señalaron cómo las ocupaciones están comprometidas, por ejemplo en cuanto a la gestión financiera. En resumen, la mirada empoderadora contribuye a la construcción de un camino hacia la inclusión. Dicha perspectiva concibe la ocupación, cultural y socialmente significativa, como una herramienta que actúa como promotora de la participación y la inclusión.

c) Una mirada sistémica: la familia como base de la intervención

En cuanto al abordaje de la familia, varios autores (Rivas et al., 2011; Boisvert et al., 2008) señalan que el terapeuta ocupacional puede ofrecer las oportunidades necesarias para que la familia pueda participar en el plan de intervención. De acuerdo con esta mirada teórica se emplean algunos instrumentos de evaluación (Rojo, 2010; Davies \& Cameron, 2010; Martin et al., 2008) propuestos desde el Modelo de Ocupación Humana (MOHO). Este modelo de TO se basa en la teoría de sistemas para explicar cómo cada ser humano organiza y ejecuta el comportamiento ocupacional (Kielhofner \& Burke, 1980). Algunos ejemplos serían la Entrevista Histórica del Funcionamiento Ocupacional, el Cuestionario Volicional, el Listado de Intereses y el Listado de Roles, entre otros (Rojo, 2010; Davies \& Cameron, 2010; Martin et al., 2008).

Asimismo, los autores muestran que orientar y asesorar a la familia es fundamental, ya que debe ser un actor más implicado en el proceso de rehabilitación y actuar como un facilitador dentro de sus entornos. Según la propuesta de Davis y Cameron (2010), el plan de trabajo de TO con la familia de personas con consumo problemático de drogas debe contribuir a que cada miembro de la familia como retome sus roles y responsabilidades. En resumen, la mirada sistémica en los estudios revisados considera a la familia un actor principal en el proceso de recuperación de las drogodependencias, ofreciendo soporte y actuando como capacitador a lo largo del plan de intervención.

\section{DISCUSIÓN}

El objetivo general de este trabajo fue actualizar, identificar y sintetizar la investigación de terapia ocupacional en el consumo problemático de drogas. Asimismo, se pretendió generar una discusión reflexiva y crítica acerca de los conocimientos actuales (ej. prioridades, perspectivas y contextos) de la TO en esta área.

Con respecto a los resultados obtenidos en este trabajo, se puede decir que algunas de las categorías 
descritas corroboran los resultados de investigaciones previas referentes al rol que se atribuye a la TO en la problemática de drogas (Díaz \& Palucci, 2010; Colomer, 2008; Thompson, 2007), la demanda de instrumentos de evaluación propios (De León et al., 2013, Rojo, 2010; Colomer et al., 2011; Rojo et al., 2011; Farías et al., 2010) y la descripción de las intervenciones de TO desde diversas perspectivas teóricas (Wasmuth, 2014; De León et al., 2013; Rojo, 2010; Rivas et al., 2011; Rojo et al., 2009; Colomer, 2008; Martin et al., 2008; Boisvert et al., 2008). Sin embargo, este trabajo de investigación aporta nueva información, puesto que las revisiones previas describía la literatura existente hasta ese momento pero no profundizaban en las diversas aproximaciones y miradas existentes desde la TO.

Así, en relación a la categoría "Contextualización" se encontró que la mayoría de los artículos procedían de España. Esta elevada producción científica, estrechamente relacionada con una mirada positivista desde las neurociencias, contrasta con la ausencia de la figura del terapeuta ocupacional como figura habitual en el ámbito de las drogodependencias. Es así como, a pesar de la elevada producción científica relativa en España, ésta parece no haber contribuido a la inclusión de la figura del TO como profesional habitual en la práctica de las drogodependencias. Esto puede ser debido a la predominancia del enfoque positivista, el cual no soporta el trabajo holístico de la TO, limitando su potencial y probable expansión profesional (ej. planes comunitarios de prevención, evaluaciones de riesgo, trabajo en escuelas y con centros penitenciarios, etc.). Por ello, se corrobora la necesidad de desarrollar estudios sobre el rol de la TO en esta área (Díaz \& Palucci, 2010; Thompson, 2007) con el objetivo de demostrar el potencial de la TO y reforzar la importancia de la incorporación de dicho profesional en el ámbito del consumo problemático de drogas desde una mirada social. Se espera que una perspectiva social y comunitaria, centrada en la inclusión de personas con consumo problemático de drogas en la comunidad, pudiese dar más coherencia al quehacer profesional y ayudar a cerrar la brecha entre la teoría actualizada y la práctica de TO en esta área.

Respecto a la categoría "Prioridades en la investigación", los intereses se corresponden con elementos básicos de la intervención, a diferencia de otras disciplinas (Espada, Gonzálvez, Orgilés Lloret, \& Guillén, 2015; Pérez, Ruano, Etminan, \& Takkouche, 2010), lo que indica que la evidencia en este ámbito podría estar en momentos iniciales de su desarrollo. Por ello, pa- rece que la profesión está tratando de asentar sus bases conceptuales en esta área, comenzando a generar evidencia por los pilares más elementales como son los instrumentos de evaluación o la valoración de la relación del desempeño ocupacional y la calidad de vida, y la descripción de las intervenciones de TO y sus resultados en esta área. Sin embargo, en este estudio se demuestra que la literatura científica existente hasta el momento no profundiza en las diversas aproximaciones y perspectivas empleadas en la investigación de TO y drogodependencias, lo cual no favorece el desarrollo teórico y práctico en esta área. Es decir, no se describe de forma detallada qué fuentes teóricas se utilizan como base para las intervenciones y/o no se justifica el uso de ciertas técnicas y/o métodos desde la teoría actualizada de TO. Asimismo, se pone de manifiesto que la población con "consumo problemático de drogas" se define de diversas maneras en la literatura, lo cual dificulta realizar una comparación entre los diferentes enfoques y tipos de intervenciones utilizadas y sus resultados. Además, se constata que existe una gran variabilidad en la terminología utilizada para hacer referencia a los aspectos ocupacionales y a las funciones que debería tener el terapeuta ocupacional a nivel práctico, lo que puede estar entorpeciendo el desarrollo de la TO en esta área. Por lo anteriormente descrito, parece que este incipiente desarrollo es confuso y sin direcciones claras. Esto puede ser debido al momento en el que se encuentra la profesión, el cual se ha caracterizado por una crítica de los pilares positivistas e individualistas de la profesión (Farías \& Laliberte Rudman, 2016; Rivas-Quarneti, 2016; Hocking, 2012; Kantartzis \& Molineux, 2011) que han limitado el rol del terapeuta ocupacional a "evaluador" en vez de agente de cambio (ej. utilizando enfoques comunitarios, facilitando la inclusión social y/o reintegración laboral de personas con consumo problemático de drogas).

En cuanto a "Miradas teóricas en la investigación" se observa una gran diversidad de perspectivas subyacentes, lo que se interpreta como un intento de las autoras por articular sus influencias teoréticas en la investigación de TO y drogodependencias, las cuales muchas veces no son explicitadas. Sin embargo, esta diversidad, además de ser una fortaleza, constituye un desafío para la profesión, ya que, en vez de construir un cuerpo de literatura sólido y basado en estudios anteriores, genera tensiones poco productivas que podrían diluir los esfuerzos para avanzar hacia un posicionamiento del TO en esta área. Cabe destacar que 
esta crítica hacia la diversidad de miradas teóricas de la investigación se enfoca en la poca conexión entre los artículos revisados, los cuales se podrían beneficiar de un diálogo crítico entre lo ya publicado y los espacios por desarrollar.

Además, esta revisión de la literatura ha demostrado una escasez de artículos que aborden la investigación desde una mirada socio-crítica o desde la CO, por lo cual se puede decir que son pocos los artículos que integran una mirada más crítica y reflexiva acerca de las direcciones que se están tomando en el área de las drogodependencias. La mayoría de los artículos son descriptivos o sistematizan las experiencias en esta área. Por lo mismo, se plantea la necesidad de avanzar en la investigación desde una mirada más crítica y reflexiva, coherente con el compromiso hacia la transformación social de la TO y con las premisas y aportaciones de la $\mathrm{CO}$ en la actualidad. Por ello, se propone profundizar en las formas de entender las drogodependencias desde la TO (ej. perspectivas, prioridades), para así reflexionar sobre las oportunidades y potencial de la TO en esta área.

\section{CONCLUSIONES}

Los resultados del presente trabajo han permitido conocer con mayor profundidad la contextualización, las prioridades y las miradas teóricas en la investigación, desde la TO, en drogodependencias. Esto ha permitido comprender las diversas percepciones de las autoras sobre la problemática del consumo de drogas. En particular, este trabajo demuestra que existe una escasa producción científica sobre TO y drogodependencias, y por ello se propone crear espacios para diálogos más reflexivos en esta área (ej. ¿Qué tipo/s de TO nos gustaría conducir y desarrollar en esta área? ¿Cuáles son los impedimentos para desarrollar una TO más comunitaria y social en el área de drogodependencias? ¿Qué desafíos y oportunidades enfrentan las/los terapeutas ocupacionales en sus prácticas diarias? ¿Qué tipo de evidencia necesitamos desarrollar y desde qué perspectivas?).

Debido a la importancia que supone la actualización teórica y práctica, la magnitud de la problemática y las diversas miradas o formas de entenderla, se propone seguir investigando empleando una visión más socio-crítica del tipo de intervenciones y perspectivas teóricas que se están utilizando y el tipo de evidencia que se está generando en esta área. Asimismo, se sugiere reflexionar sobre la posibilidad de una potencial mayor contribución de la TO y la CO hacia el colectivo aproximado desde este posicionamiento, en especial desde los contextos socioeconómicos de cada país. Se espera con los resultados de este trabajo, así como con futuras investigaciones, poder contribuir a una práctica basada en los movimientos teóricos contemporáneos, lo que en última instancia se considera que favorecerá potencialmente la inclusión de la TO en el ámbito del consumo problemático de drogas.

\section{REFERENCIAS BIBLIOGRÁFICAS}

Arksey, H. \& O'Malley, L. (2005). Scoping studies: Towards a methodological framework. International Journal of Social Research Methodology, 8(1), 19-32. Doi: 10.1080/1364557032000119616.

Boisvert, R.A., Martin, L.M., Grosek, M. \& Clarie, A.J. (2008). Effectiveness of a peer-support community in addiction recovery: Participation as intervention. Occupational Therapy International Journal, 15(4), 205-20. Doi: 10.1002/oti.257.

Colomer, M.C., Cabellos, A.I. \& Bustos, M.A. (2011). Desempeño ocupacional e importancia de las AVD en el tratamiento de personas adictas. XXXVIII Jornadas Nacionales de "Socidrogalcohol", 52. Recuperado de http://www.codajic.org/sites/www.codajic.org/ files/Socidrogalcohol\%20Jornadas\%202011.pdf.

Colomer, M.C. (2008). Intervención del Terapeuta Ocupacional con población drogodependiente. Revista Electrónica de Terapia Ocupacional de Galicia, 1(3). Recuperado de https://dialnet.unirioja.es/ servlet/articulo?codigo=2938192.

Consellería de Sanidade e Servicio Galego de Saúde - SERGAS (2011). Plan de trastornos adictivos de Galicia 2011-2016. Santiago de Compostela, España. Recuperado de http://xuventude.xunta.es/ uploads/docs/Observatorio/Plan_de_trastornos_adictivos_de_ Galicia_2011-2016.pdf.

Davies, R. \& Cameron, J. (2010). Self-identified occupational competencies, limitations and priorities for change in the occupational lives of people with drug misuse problems. British Journal of Occupational Therapy, 73(6), 251-260. Doi: 10.4276/030802210X127599 25468907.

De León Rodríguez, D., Cantero Garlito, P.A. \& Zabala Baños, M. (2013). Desempeño ocupacional y calidad de vida en personas con adicción a sustancias. Revista Electrónica de Terapia Ocupacional de Galicia, 10(18), 21. Recuperado de http://www.revistatog.com/ num18/pdfs/original1.pdf. 
Degenhardt, L., Hall, W., Warner-Smith, M. \& Lynskey, M. (2004). Illicit drug use. En M. Ezzati, A. D. Lopez, A. Rodgers \& C. J. L. Murray (Eds.), Comparative quantification of health risks: Global and regional burden of disease attributable to selected major risk factors (Vol. 1, pp. 1109-1176). Geneva: World Health Organization. Recuperado de http://apps.who.int/iris/bitstream/10665/42792/1/9241580348_eng_Volume1.pdf.

Díaz, L.P. \& Palucci, M.H. (2010). El papel de los profesionales en centros de atención en drogas en ambulatorios de la ciudad de Bogotá, Colombia. Revista latino-americana de enfermagem, 18, 57381. Doi: 10.1590/S0104-11692010000700013.

Espada, J.P., Gonzálvez, M., Orgilés, M., Lloret, D. \& Guillén, A. (2015). Meta-analysis of the effectiveness of school substance abuse prevention programs in Spain. Psicothema, 27(1), 5-12. Doi: 10.7334/ psicothema2014.106.

Farías, L., Guerra, V., Cifuentes, T., Rozas, S. \& Riveros, Mª E. (2010). Consumo problemático de drogas y Terapia Ocupacional: Componentes ocupacionales evaluados durante el proceso de tratamiento y rehabilitación. Revista Chilena de Terapia Ocupacional, 10, 45-56. Doi: 10.5354/0717-5346.2010.10559.

Farías, L. \& Laliberte Rudman, D. (2016). A critical interpretive synthesis of the uptake of critical perspectives in occupational science. Journal of Occupational Science, 23(1), 33-50. Doi: 10.1080/14427591.2014.989893.

González, T. \& Cano, A. (2010). Introducción al análisis de datos en investigación cualitativa: Tipos de análisis y proceso de codificación (II) Nure Investigación, 45, 1-10. Recuperado de http://www.nureinvestigacion.es/OJS/index.php/nure/article/view/485/474.

Hocking, C. (2012). Occupations through the looking glass: Reflecting on occupational scientists' ontological assumptions. En G. E. Whiteford \& C. Hocking (Eds.), Occupational science: Society, inclusion, participation (pp. 54-66). West Sussex, UK Wiley-Blackwell.

Kantartzis, S. \& Molineux, M. (2011). The influence of Western society's construction of a healthy daily life on the conceptualisation of occupation. Journal of Occupational Science, 18(1). Doi:10.1080/144 27591.2011.566917.

Kielhofner, G. (2008). The environment and human occupation. En Kielhofner, G. (ed.), A model of human occupation: Theory and application (4th ed., pp. 85-97). Baltimore, MD: Lippincott Williams \& Wilkins

Kielhofner, G. \& Burke, J.P. (1980). A model of human occupation, Part 1. Conceptual framework and content. American Journal of Occupational Therapy, 34, 572-581. Doi: 10.5014/ajot.34.9.572.

Kronenberg, F., Simó, S. \& Pollard, N. (2007). Terapia ocupacional sin fronteras: Aprendiendo del espíritu de los sobrevivientes. Madrid: Editorial Médica Panamericana.
Law, M., Stewart, D., Lette, I., Pollock, N., Bosch, J. \& Westmorland, M. (1998). Instrucciones para el formulario de revisión crítica de estudios cualitativos. McMaster University. Traducción: TO Mariela Nabergoi. Recuperado de http://srs-mcmaster.ca/wp-content/ uploads/2015/04/Guidelines-for-Critical-Review-Form-Qualitative-Studies-Spanish.pdf.

Lee, S., Powell, N.J. \& Esdaile, S. (2001). A functional model of cognitive rehabilitation in occupational therapy. Canadian Journal Occupational Therapy, 68(1), 41-50. doi:10.1177/000841740106800105.

Martin, L., Bliven, M. \& Boisvert, R. (2008). Occupational performance, self-esteem, and quality of life in substance addictions recovery. OTJR: Occupation, Participation and Health, 28(2), 81-88. Doi: 10.3928/15394492-20080301-05.

Ministerio de Sanidad y Política Social (2012). Informe Nacional Social de España. Madrid: Delegación del Gobierno del Reino de España. Recuperado de http://www.msssi.gob.es/ssi/familiasInfancia/inclusionSocial/inclusionSocialEspana/programasNacionales/docs/informeNacionalSocial2012.pdf.

Observatorio Europeo de las Drogas y las Toxicomanías (EMCDDA). (2013). Informe Europeo sobre Drogas. Doi: 10.2810/882.

Organización Mundial de la Salud (2012). Hay que mejorar el acceso de los drogodependientes a la atención sanitaria. Organización Mundial de la Salud. Recuperado de http://www.who.int/mediacentre/ news/notes/2012/drug_use_20120626/es/.

Pérez, M., Ruano, A., Etminan, M. \& Takkouche, B. (2010). Un meta análisis de la exposición a polvo de madera y el riesgo de asma. Archivos de prevención de riesgos laborales, 13(1), 44-45. Recuperado de http://www.archivosdeprevencion.com/view_document. php?tpd=2\&i=1929.

Pollard, N., Sakellariou, D. \& Kronenberg, F. (2008). A political practice of occupational therapy. Edinburgh: Elsevier Science.

Rivas-Quarneti, N. (2016). Estudio de las ocupaciones cotidianas para la promoción de la salud de las mujeres inmigrantes en situación de vulnerabilidad (Tesis doctoral). Universidade da Coruña, España. Recuperado de http://hdl.handle.net/2183/16259.

Rivas-Quarneti, N., Gómez-Sánchez, T., Movilla-Fernández, M. \& Viana-Moldes, I. (2011). Tecnologías de la información y la comunicación (TIC) y la promoción de la participación. Revista Electrónica de Terapia Ocupacional de Galicia, 8(7), 43-56. Recuperado de http://www.revistatog.com/suple/num7/tic.pdf.

Rojo, G. (2010). Tratamiento de terapia ocupacional en adicciones: enfoque neurocientífico. Terapia Ocupacional: Revista informativa de la Asociación Profesional Española de Terapeutas Ocupacionales, 53, 36. Recuperado de http://dialnet.unirioja.es/servlet/ articulo?codigo $=3392138$. 
Rojo, G., Pedrero, E.J., Ruiz, J.M., Llanero, M. \& Puerta, C. (2011). Evaluación del desempeño ocupacional en la vida cotidiana en adictos. Creación de un instrumento de medida: el ADO. Adicciones: Revista de socidrogalcohol, 23(1), 27-35. Doi: 10.20882/ adicciones.164.

Rojo, G., Pedrero, E.J., Ruiz, J.M., Llanero, M., Puerta, C. \& Olivar, A. (2009). Terapia Ocupacional en la rehabilitación de la disfunción ejecutiva en adictos a sustancias. Trastornos adictivos: Órgano Oficial de la Sociedad Española de Toxicomanías, 11(2), 96-105. Doi: 10.1016/S1575-0973(09)72057-4.

Taylor, S. \& Bogdan, R. (1990). Introducción a los métodos cualitativos de investigación. Barcelona: Paidós.

Thompson, K. (2007). Occupational therapy and substance use disorders: Are practitioners addressing these disorders in practice? Occupational Therapy in Health Care, 21(3), 61-77. Doi: 10.1080/ J003v21n03_04.

United Nations Office for Drug Control and Crime Prevention (UNDCP). (2002). Global Illicit Drug Tends. United Nations Office for Drug Control and Crime Prevention. Recuperado de https://www.unodc.org/pdf/report_2002-06-26_1/report_2002-06-26_1.pdf.

Wasmuth, S., Crabtree, J. \& Scott, P. (2014). Exploring addiction-as-occupation. British Journal of Occupational Therapy, 77(12), 605-613. Doi: 10.4276/030802214X14176260335264.

World Health Organization (WHO). (2002). Management of substance abuse. The global Burden. World Health Organization. Recuperado de http://www.who.int/substance_abuse/facts/global_burden/en/. 